\title{
Wideband Circularly Polarized Dielectric Rod Antenna
}

\author{
Min Guo, ${ }^{1,2}$ Ji-Jun Yan, ${ }^{2}$ Shun-Shi Zhong, ${ }^{1}$ and Zhu Sun ${ }^{1}$ \\ ${ }^{1}$ School of Communication and Information Engineering, Shanghai University, Shanghai 200072, China \\ ${ }^{2}$ Shanghai Radio Equipment Research Institute, Shanghai 200438, China
}

Correspondence should be addressed to Min Guo, minguol@163.com

Received 16 January 2012; Revised 9 April 2012; Accepted 22 April 2012

Academic Editor: Hamsakutty Vettikalladi

Copyright () 2012 Min Guo et al. This is an open access article distributed under the Creative Commons Attribution License, which permits unrestricted use, distribution, and reproduction in any medium, provided the original work is properly cited.

\begin{abstract}
A new dielectric rod antenna (DRA) is introduced to produce circular polarization (CP) over a wide frequency band without a complex feed network. Along with the simulated results, measured results of the antenna prototype are presented, showing a $3 \mathrm{~dB}$ axial ratio (AR) CP bandwidth of $17.7 \%$. The radiation characteristics of the fabricated antenna are also demonstrated showing the measured gain of better than $6.2 \mathrm{dBi}$. Moreover, the measured impedance bandwidth (VSWR $\leq 2$ ) reaches $20.1 \%$, from $8.75 \mathrm{GHz}$ to $10.7 \mathrm{GHz}$, while the $\mathrm{CP}$ beamwidth $(\mathrm{AR} \leq 3 \mathrm{~dB})$ at the central frequency is measured over $120^{\circ}$.
\end{abstract}

\section{Introduction}

Circularly polarized waves are often used to offer better propagation characteristics through the atmosphere and reduced multipath effect as well as the flexibility of orientation between the transmitting and receiving antennas. Satellite CP systems require antennas exhibiting an excellent axial ratio over a wide frequency band and over a wide beamwidth to realize low power signal reception at low elevation angle. The $\mathrm{CP}$ approach includes two categories: single feed and multipoint feed. The single feed is of simplicity advantage of not requiring an external polarizer such as $90^{\circ}$ hybrid coupler, but it usually leads to a limited AR bandwidth in the range of a few percentage. For instance, a single-fed elliptical dielectric resonator antenna is proposed with a circular polarization bandwidth of $3.5 \%$ [1]. In [2], a rectangular dielectric resonator antenna outer-fed by a square spiral strip provides CP operation over a broad CP bandwidth of $14 \%$ with an impedance bandwidth of $11 \%$. The multipoint feed is of a wider CP bandwidth, but an external feeding circuit is required.

The surface wave antenna, employing a dielectric rod, is popular because of its wide bandwidth, agility in controlling the radiation pattern shape, ease of fabrication and low cost $[3,4]$. In lower microwave range, this antenna may be used as a feed system for reflector antennas [5] and at the millimeter wave range, it can be integrated directly with the monolithic integrated circuits $[6,7]$. In recent years, ultrawideband (UWB), dual-band, and dual-polarized dielectric rod antennas have been investigated [8-10]. A number of theoretical and experimental studies [11] have been carried out to understand the radiation mechanism with linearly polarized operation. Lately, more attentions have been paid to the circularly polarized DRA due to its wider applications, especially in the satellite communication systems.

In this paper a single-fed CP DRA is presented, where the phase transformer is made by introducing a dielectric sheet in the circular feed waveguide. This CP design does not require a complex feed network or special configuration of the radiating element. The measured results show a circular polarization bandwidth of $17.7 \%$, which is greater than the bandwidths reported in the literature for singlefed CP DRAs. Reasonable agreement between the simulation and the measurement is obtained. Details of the proposed antenna design are described and experimental results of its $\mathrm{CP}$ performance are presented and discussed.

\section{Antenna Design}

The geometry of proposed DRA is shown in Figure 1. The diameter $a_{1}$ of the feed waveguide is mainly determined by the cutoff frequency of $\mathrm{TE}_{11}$ mode and is chosen as $16.4 \mathrm{~mm}$ to operate at X-band with a frequency range of $9.0 \mathrm{GHz}$ to $11.5 \mathrm{GHz}$. In the circular feed waveguide, a centered 


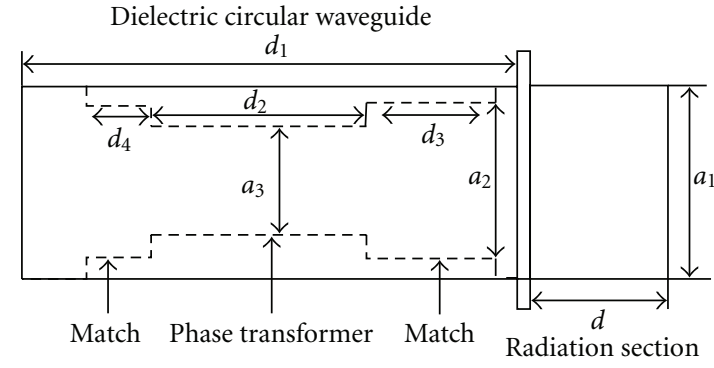

(a) side view

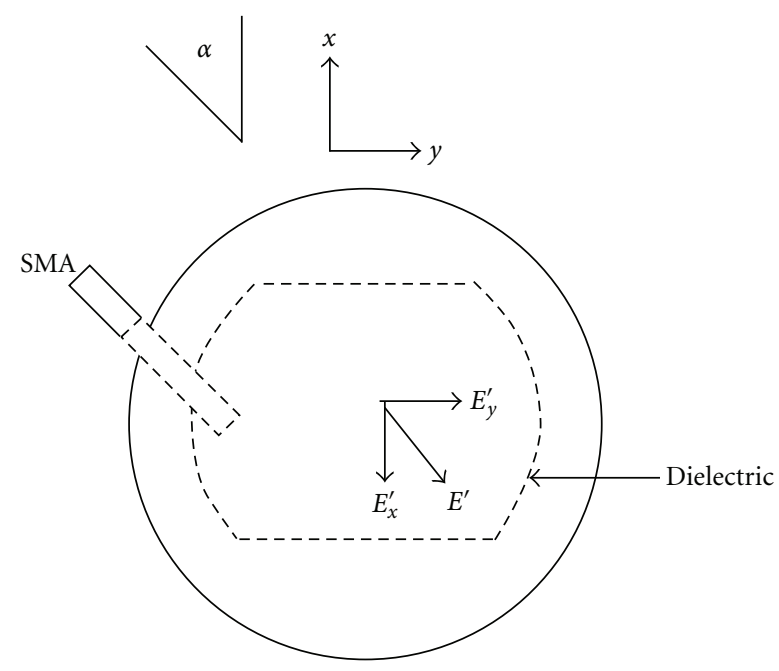

(b) bottom view

FIGURE 1: Geometry of dielectric rod antenna.

dielectric sheet with relative permittivity $\varepsilon_{r}=2.05$ is located, which consists of a phase-shift segment and two matching segments in two ends. To produce CP radiation, the dielectric rod should be excited by two orthogonal electric field components, $\vec{E}_{x}$ and $\overrightarrow{E_{y}}$, with equal amplitude and 90-degree phase difference. The probe of coaxial feed is oriented by $45^{\circ}$ to form two orthogonal electric field components with equal amplitude, as shown in the Figure 1(b), which is for LHCP operation, while for RHCP operation the probe in the other diagonal is required.

Based on the microwave waveguide theory, the phase difference between two orthogonal electric field components $\overrightarrow{E_{x}}$ and $\overrightarrow{E_{y}}$ is given by

$$
\Delta \phi=\frac{2 \pi L \sqrt{\varepsilon_{r x}-\left(\lambda_{0} / \lambda_{c}\right)^{2}}-\sqrt{\varepsilon_{r y}-\left(\lambda_{0} / \lambda_{c}\right)^{2}}}{\lambda_{0}},
$$

where $L$ is the length of dielectric sheet, $\lambda_{0}$ is the free-space wavelength, and $\lambda_{c}$ is the cut-off wavelength. The effective relative permittivity of $y$ direction is

$$
\varepsilon_{r y}=\varepsilon_{r} .
$$

The effective relative permittivity of $x$ direction is

$$
\varepsilon_{r x}=1+\frac{\left(\varepsilon_{r}-1\right) A_{1}}{A_{0}}
$$

where $A_{1}$ is the cross area of the circular waveguide and $A_{0}$ is the cross area of the dielectric sheet.

\section{Experimental Results}

The proposed antenna prototype has been fabricated and measured to verify its $\mathrm{CP}$ operation, whose parameters are optimized by using the simulation software HFSS 11



FIGURE 2: Simulated and measured VSWR characteristics.

with the final optimized parameters: $a_{1}=16.4 \mathrm{~mm}, a_{2}=$ $12.2 \mathrm{~mm}, a_{3}=9.6 \mathrm{~mm}, d=13.3 \mathrm{~mm}, d_{1}=73.1 \mathrm{~mm}, d_{2}=$ $34 \mathrm{~mm}, d_{3}=6.8 \mathrm{~mm}$, and $d_{4}=6.8 \mathrm{~mm}$. Figure 2 displays the simulated and measured VSWR curves of the antenna. The measured impedance bandwidth is $20.1 \%$ for VSWR less than 2, covering the frequency range from $8.75 \mathrm{GHz}$ to $10.7 \mathrm{GHz}$. Figure 3 shows the simulated and measured axial ratio against frequency and angle. The simulated CP bandwidth of $3 \mathrm{~dB}$ axial ratio is $2.2 \mathrm{GHz}$ or $22.8 \%$ with the minimum $\mathrm{AR}$ of $0.29 \mathrm{~dB}$ at $11.1 \mathrm{GHz}$. The measured $3 \mathrm{~dB}$ axial-ratio $\mathrm{CP}$ bandwidth is $2.75 \mathrm{GHz}$ or about $17.7 \%$ with the central frequency $9.875 \mathrm{GHz}$, which may be the widest one for single-fed CP DRAs in the open literature. The difference between simulated and measured bandwidths is probably due to the tolerance of DRA permittivity and 




(a) axial ratio against frequency

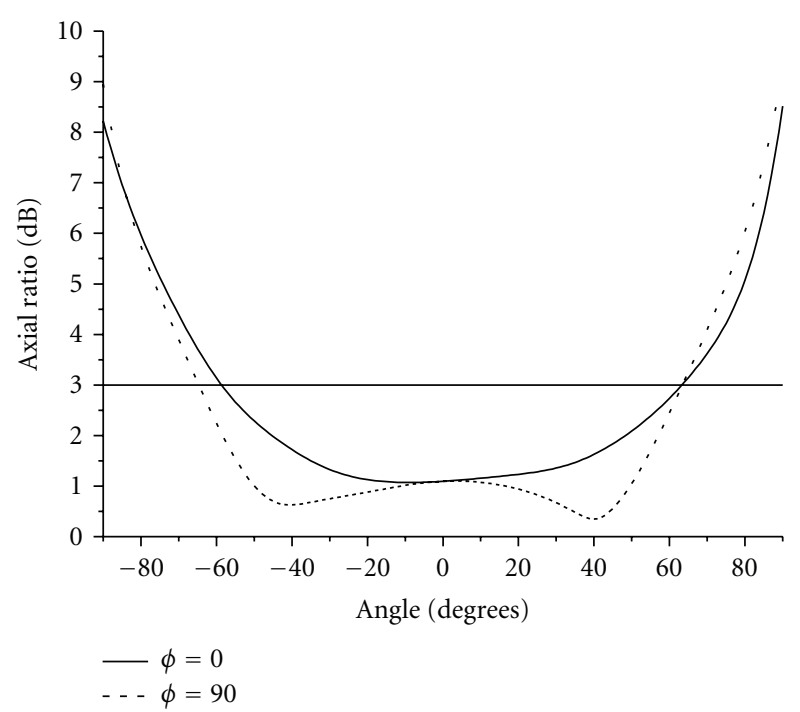

(b) axial ratio against angle

FIgURE 3: Simulated and measured axial ratio.

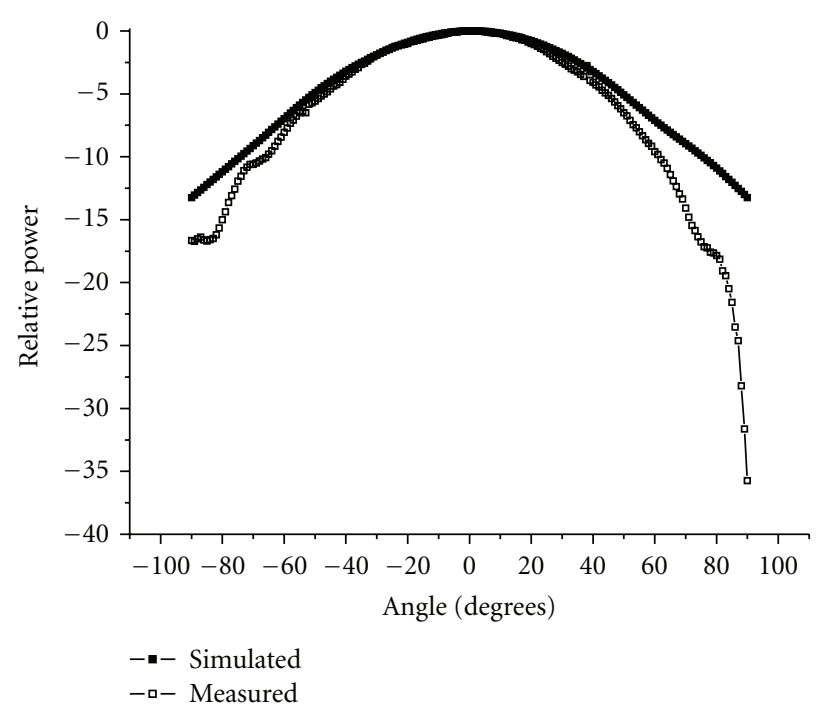

(a) $x$-z plane

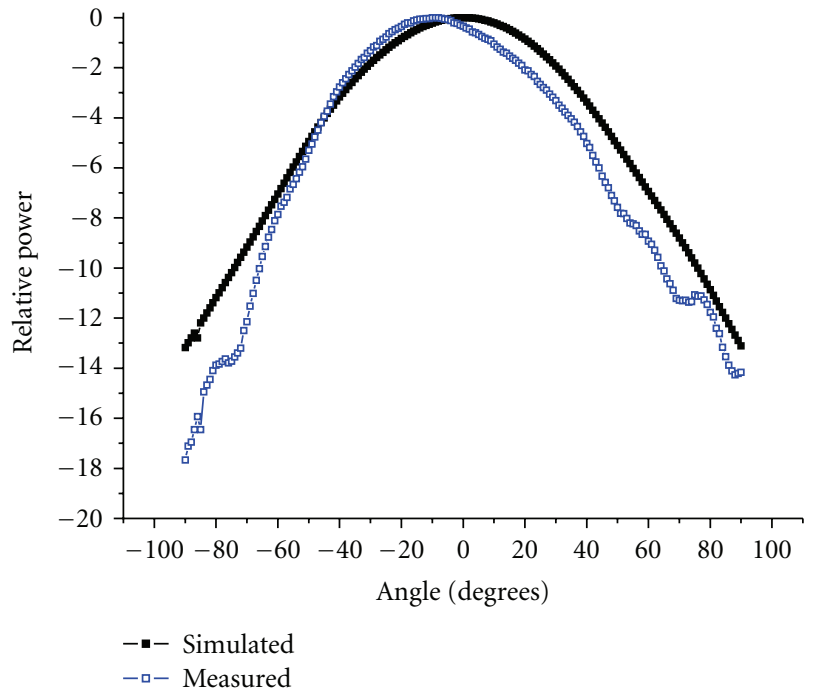

(b) $y-z$ plane

FIGURE 4: Simulated and measured radiation patterns at central frequency.

dimensions. Figure 4 displays simulated and measured radiation patterns at central frequency, showing fine agreement. The measured half-beamwidth in $x-z$ plane is $69.66^{\circ}$, and the half-beamwidth in $y-z$ plane is $68.87^{\circ}$. The measured antenna gain is over $6.2 \mathrm{dBi}$ and approximately constant over a wide bandwidth, as shown in Figure 5. The CP beamwidth $(\mathrm{AR} \leq 3 \mathrm{~dB})$ at central frequency is measured over $120^{\circ}$.

\section{Conclusion}

A new single-fed DRA without a 90-degree hybrid coupler has been introduced to realize a wide CP bandwidth. The
CP operation is easily obtained by controlling the length and width of the dielectric sheet in the circular feed of waveguide. The antenna is compact in structure, because, that it consists of only a cylindrical DRA with radius of $16.4 \mathrm{~mm}$, length of $75 \mathrm{~mm}$, and a feed waveguide. The measured CP bandwidth is $17.7 \%$ with a measured axial ratio minimum of $0.869 \mathrm{~dB}$ and a $\mathrm{CP}$ beam width of $120^{\circ}$. The proposed antenna exhibits an impedance bandwidth of $20.1 \%$ around the same frequency range. With these features, this antenna is attractive for the satellite communication applications. 


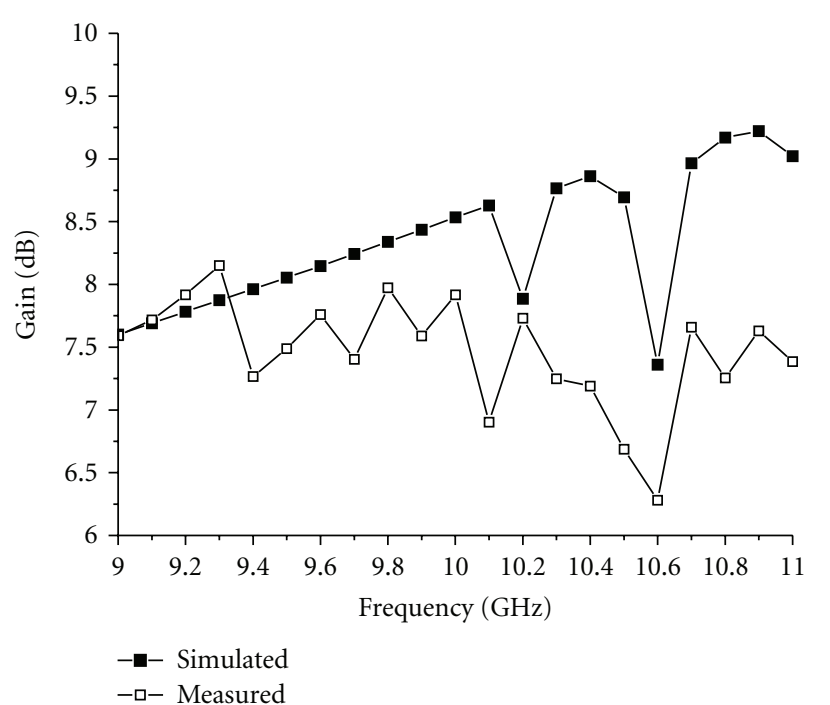

FIgURE 5: Simulated and measured gains at $\theta=\varphi=0^{\circ}$.

\section{Acknowledgments}

This work was supported by the National Natural Science Foundation of China under Grant no. 61171031 and the Shanghai Leading Academic Discipline Project under Grant no. S30108.

\section{References}

[1] A. A. Kishk, "An elliptic dielectric resonator antenna designed for circular polarization with single feed," Microwave and Optical Technology Letters, vol. 37, no. 6, pp. 454-456, 2003.

[2] M. I. Sulaiman and S. K. Khamas, "A singly fed rectangular dielectric resonator antenna with a wideband circular polarization," IEEE Antennas and Wireless Propagation Letters, vol. 9, pp. 615-618, 2010.

[3] C. Kumar, V. V. Srinivasan, V. K. Lakshmeesha, and S. Pal, "Design of short axial length high gain dielectric rod antenna," IEEE Transactions on Antennas and Propagation, vol. 58, no. 12, pp. 4066-4069, 2010.

[4] S. M. Hanham and T. S. Bird, "High efficiency excitation of dielectric rods using a magnetic ring current," IEEE Transactions on Antennas and Propagation, vol. 56, no. 6, pp. 1805-1808, 2008.

[5] K. H. Lee, C. C. Chen, and R. Lee, "UWB dual-linear polarization dielectric horn antennas as reflector feeds," IEEE Transactions on Antennas and Propagation, vol. 55, no. 3, pp. 798-804, 2007.

[6] H. Zhou, X. Chen, D. S. Espinoza, A. Mickelson, and D. S. Filipovic, "Nanoscale optical dielectric rod antenna for on-chip interconnecting networks," IEEE Transactions on Microwave Theory and Techniques, 2011.

[7] M. Sun, Y. P. Zhang, G. X. Zheng, and W. Y. Yin, "Performance of intra-chip wireless interconnect using on-chip antennas and UWB radios," IEEE Transactions on Antennas and Propagation, vol. 57, no. 9, pp. 2756-2762, 2009.

[8] M. Leib, A. Vollmer, and W. Menzel, "An ultra-wideband dielectric rod antenna fed by a planar circular slot," IEEE Transactions on Microwave Theory and Techniques, vol. 59, no. 4, pp. 1082-1089, 2011.
[9] G. Adamiuk, T. Zwick, and W. Wiesbeck, "Compact, dualpolarized UWB-antenna, embedded in a dielectric," IEEE Transactions on Antennas and Propagation, vol. 58, no. 2, pp. 279-286, 2010.

[10] D. Yaghjian and E. T. Kornhauser, "A model analysis of the dielectric rod antenna excited by the HE11 mode," IEEE Transactions on Antennas and Propagation, vol. 20, pp. 122128, 1972.

[11] J. Y. Chung and C. C. Chen, "Two-layer dielectric rod antenna," IEEE Transactions on Antennas and Propagation, vol. 56, no. 6, pp. 1541-1547, 2008. 

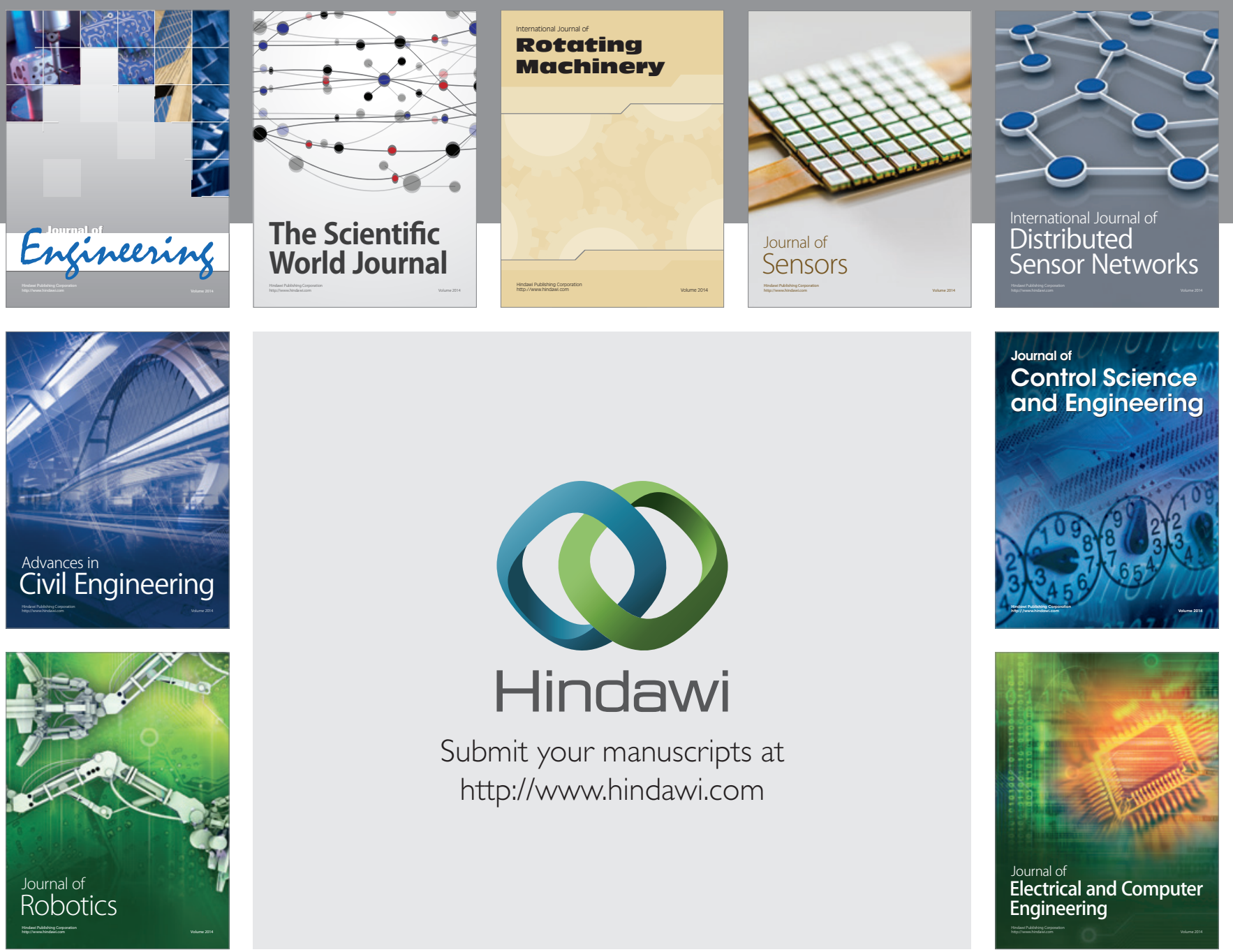

Submit your manuscripts at

http://www.hindawi.com
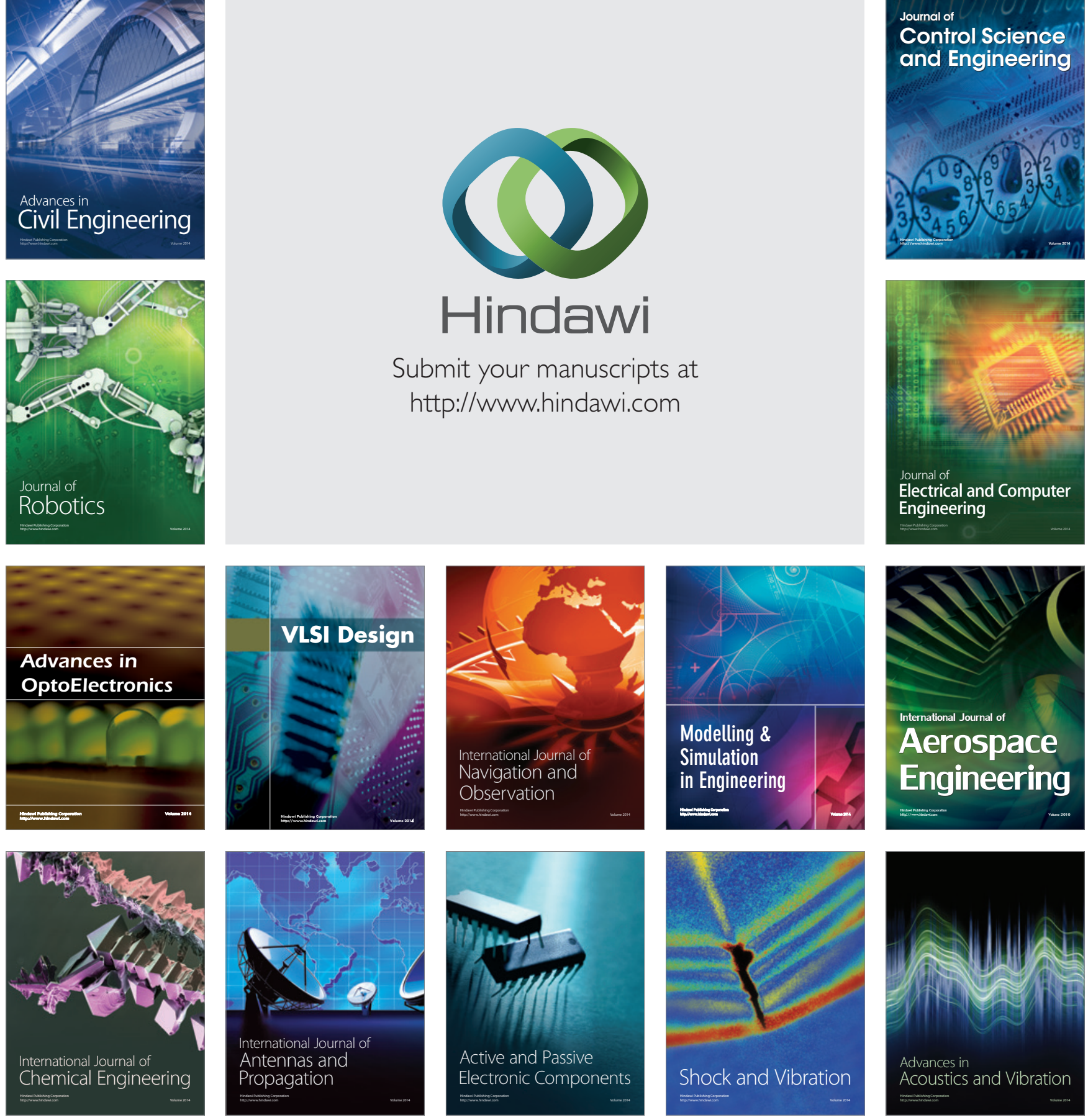